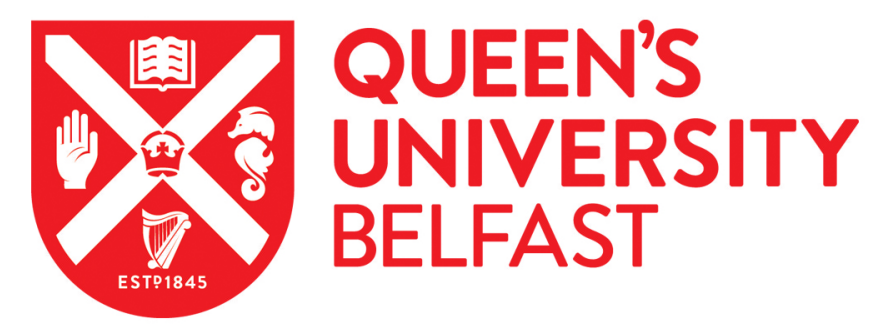

\title{
Single- and two-mode quantumness at a beam splitter
}

Brunelli, M., Benedetti, C., Olivares, S., Ferraro, A., \& Paris, M. G. A. (2015). Single- and two-mode

quantumness at a beam splitter. Physical Review A (Atomic, Molecular, and Optical Physics), 91(6), [062315]. https://doi.org/10.1103/PhysRevA.91.062315

Published in:

Physical Review A (Atomic, Molecular, and Optical Physics)

Document Version:

Peer reviewed version

Queen's University Belfast - Research Portal:

Link to publication record in Queen's University Belfast Research Portal

Publisher rights

(C) 2015 American Physical Society

\section{General rights}

Copyright for the publications made accessible via the Queen's University Belfast Research Portal is retained by the author(s) and / or other copyright owners and it is a condition of accessing these publications that users recognise and abide by the legal requirements associated with these rights.

Take down policy

The Research Portal is Queen's institutional repository that provides access to Queen's research output. Every effort has been made to ensure that content in the Research Portal does not infringe any person's rights, or applicable UK laws. If you discover content in the Research Portal that you believe breaches copyright or violates any law, please contact openaccess@qub.ac.uk. 


\title{
Single- and two-mode quantumness at a beam splitter
}

\author{
Matteo Brunelli* \\ Centre for Theoretical Atomic, Molecular and Optical Physics, School of Mathematics and Physics, \\ Queen's University Belfast, Belfast BTr1NN, United Kingdom \\ Claudia Benedetti ${ }^{\dagger}$ \\ Dipartimento di Fisica, Università degli Studi di Milano, I-20133 Milano, Italy \\ Stefano Olivares $\ddagger$ \\ Dipartimento di Fisica, Università degli Studi di Milano, I-20133 Milano, Italy and \\ CNISM UdR Milano Statale, I-20133 Milano, Italy \\ Alessandro Ferraro ${ }^{\S}$ \\ Centre for Theoretical Atomic, Molecular and Optical Physics, School of Mathematics and Physics, \\ Queen's University Belfast, Belfast BT71NN, United Kingdom \\ Matteo G. A. Paris $₫$ \\ Dipartimento di Fisica, Università degli Studi di Milano, I-20133 Milano, Italy and \\ CNISM UdR Milano Statale, I-20133 Milano, Italy
}

(Dated: February 24, 2015)

\begin{abstract}
In the context of bipartite bosonic systems, two notions of classicality of correlations can be defined: $P$-classicality, based on the properties of the Glauber-Sudarshan $P$-function; and $C$ classicality, based on the entropic quantum discord. It has been shown that these two notions are maximally inequivalent in a static (metric) sense - as they coincide only on a set of states of zero measure. We extend and reinforce quantitatively this inequivalence by addressing the dynamical relation between these types of non-classicality in a paradigmatic quantum-optical setting: the linear mixing at a beam splitter of a single-mode Gaussian state with a thermal reference state. Specifically, we show that almost all $P$-classical input states generate outputs that are not $C$-classical. Indeed, for the case of zero thermal reference photons, the more $P$-classical resources at the input the less $C$-classicality at the output. In addition, we show that the $P$-classicality at the input - as quantified by the non-classical depth — does instead determine quantitatively the potential of generating output entanglement. This endows the non-classical depth with a new operational interpretation: it gives the maximum number of thermal reference photons that can be mixed at a beam splitter without destroying the output entanglement.
\end{abstract}

PACS numbers: 03.67.Mn, 42.50.Dv

\section{INTRODUCTION}

Since the early days of quantum mechanics considerable efforts have been spent in establishing whether a given physical system possesses genuinely quantum features. As far as bosonic systems are concerned, Wigner first attacked this problem introducing a quantum analogue to the classical phase-space [1]. Later on, a systematic approach was finally developed in the framework of quantum optics, with the introduction of various classes of quasi-probability distributions defined over the quantum phase-space. Specifically, the analytical features of such distributions unveil physical constraints: when-

\footnotetext{
*Electronic address: mbrunelli01@qub.ac.uk

${ }^{\dagger}$ Electronic address: claudia.benedetti@unimi.it

$\ddagger$ Electronic address: stefano.olivares@mi.infn.it

$\S$ Electronic address: a.ferraro@qub.ac.uk

IElectronic address: matteo.paris@fisica.unimi.it
}

ever the normally-ordered distribution function - called Glauber-Sudarshan $P$-function $[2,3]$ - behaves like a regular probability distribution, the corresponding state can be described as a statistical ensemble of classical fields and, in this sense, it cannot show any non-classical feature [4]. In the following, these states will be referred to as $P$-classical states.

On the other hand, the more recent development of quantum information theory promoted a reconsideration of the quantumness of physical systems from an information-theoretical perspective. Since quantum systems can be correlated in ways unaccessible to classical ones, the discrimination between classical and nonclassical states of a given system is pursued by studying the nature of the correlations among its subparts. In particular, quantum entanglement accounts for quantum correlations that may lead to the violation of local realism [5]. Moreover, even separable (i.e., non-entangled) states have been recognized to retain non-classical features, leading to the introduction of an entropic measure, called quantum discord, to capture the quantum features 
of correlations beyond entanglement [6]. Following this criterion, classical states can be defined as states with vanishing discord, and we will refer to this notion as $C$ classicality.

Although both acceptable and well-grounded, the two foregoing notions of classicality have been shown to be radically different, indeed maximally inequivalent, in the following sense: only a zero-measure set of states is classical according to both criteria [7]. Besides embodying a matter of fundamental interest, this conclusion is also relevant for practical purposes, since it enlightens different resources in quantum information processing [8]. However, such a characterization is based on purely geometrical considerations and, as a consequence, it is intrinsically static. In particular, the relation between $C$ and $P$-classicality in common physical processes remains unclear. In addition, a quantitative comparison between these two notions in terms of their respective figures of merit still lacks.

In this work, we address the above issues in the context of quantum optics, whose description in term of the phase-space offers a natural framework to develop a quantitative analysis [9]. A paradigmatic setting in quantum optics is constituted by Gaussian states and operations, due to their relevance for quantum technologies and their thorough theoretical characterization [10-12]. Specifically, we address the dynamical relation of $P$ - and $C$-nonclassical states arising from the linear mixing of Gaussian states at a beam splitter. In this setting, the availability of analytical expressions to quantify Gaussian P-classicality - in terms of the non-classical depth - and Gaussian discord and entanglement, is crucial to work out a quantitative comparison between the various notions of non-classicality.

In particular, we consider the mixing of a generic Gaussian state with a reference thermal state, and explore the relationships between the classicality of the input state and the $P$ - and $C$-classicality at the output. Specifically, we show that almost all $P$-classical input states generate output states that are not $C$-classical. Indeed, for the case of zero thermal reference photons, the more $P$ classical resources at the input the less $C$-classicality at the output. These findings strengthen the inequivalence between $P$ - and $C$-classicality by quantitatively extending it to a process in which correlations are dynamically generated, rather then statically analyzed as in Ref.[7]. In addition, we show that the $P$-classicality at the input does instead determine quantitatively the potential of generating output entanglement. This endows the nonclassical depth with a new operational interpretation: it gives the maximum number of thermal reference photons that can be mixed at a beam splitter without destroying the output entanglement.

The paper is structured as follows. In Section II we give a brief account on Gaussian states and their phasespace representation, focusing on their bilinear interaction in linear optical devices. In Section III we review the two notions of nonclassicality and establish the notation for Gaussian discord, non-classical depth and entanglement used in the following. The reader familiar with the foregoing topic can skip the respective sections. In Section IV we analyze in details the generation of $P$ - and $C$-nonclassicality by mixing of a Gaussian state with the vacuum, whereas in Section $V$ we focus attention to the mixing with a thermal state, also introducing the concept of effective nonclassicality. Section VI closes the paper with some concluding remarks.

\section{LINEAR MIXING OF GAUSSIAN STATES}

The simplest bilinear interaction involving two bosonic field modes described by the annihilation operators $\hat{a}_{1}$ and $\hat{a}_{2}\left(\right.$ with $\left.\left[\hat{a}_{k}, \hat{a}_{k}^{\dagger}\right]=\hat{\mathbb{I}}\right)$ corresponds to the mode mixing and it is described by an effective Hamiltonian of the form $H_{I} \propto\left(\hat{a}_{1}^{\dagger} \hat{a}_{2}+\hat{a}_{1} \hat{a}_{2}^{\dagger}\right)$. This kind of interaction is very common in different quantum systems, ranging from optical modes in linear optical devices [13] to collective modes in ultracold atoms [14], opto- and nanomechanical oscillators [15-18] and superconducting resonators $[19,20]$. For the sake of clarity, in this paper we focus on the quantum optics realm and we address the correlations properties of the two optical modes emerging from a beam splitter (BS) when the input ones are excited in Gaussian states.

Gaussian states (GSs) are states with Gaussian Wigner functions [21] and an exhaustive information about them is provided by the knowledge of the first and second statistical moments of the quadrature operators. Information about correlations is contained in the second moment and from now on we set the first moments to zero, without loss of generality. Upon introducing the quadrature operators $\hat{q}=\left(\hat{a}+\hat{a}^{\dagger}\right) / \sqrt{2}$ and $\hat{p}=\left(\hat{a}-\hat{a}^{\dagger}\right) /(i \sqrt{2})$, the covariance matrix (CM) of a single-mode GS of $\hat{a}$ is defined as $[\boldsymbol{\sigma}]_{k l}=\frac{1}{2}\left\langle\left\{R_{k}, R_{l}\right\}\right\rangle-\left\langle R_{k}\right\rangle\left\langle R_{l}\right\rangle$, with $k, l=1,2$, being $\mathbf{R}^{T}=\left(R_{1}, R_{2}\right) \equiv(\hat{q}, \hat{p})$ the vector of the quadratures and $\{\cdot, \cdot\}$ the anticommutator. Now, the canonical commutation relations take the form $\left[R_{k}, R_{l}\right]=i \omega_{k l}$, where $\omega_{k l}=\left(1-\delta_{k l}\right)(-1)^{l}$ are the entries of the is the $2 \times 2$ symplectic form $\boldsymbol{\omega}$. The set of the eigenvalues $(q, p) \in \mathbb{R}^{2}$ of the position and momentum-like operators, endowed with the symplectic form $\boldsymbol{\omega}$, spans the real symplectic space $\Gamma=\left(\mathbb{R}^{2}, \boldsymbol{\omega}\right)$, which is referred to as the phase space of the mode $\hat{a}_{1}$. A single-mode GS may always be written as

$$
\varrho_{1}\left(n_{t}, n_{s}\right)=S(r) \nu\left(n_{t}\right) S^{\dagger}(r),
$$

where $S(r)=\exp \left[\frac{1}{2}\left(r a^{\dagger 2}-r^{*} a^{2}\right)\right], r \in \mathbb{C}$, is the squeezing operator and

$$
\nu\left(n_{t}\right)=\left(n_{t}+1\right)^{-1}\left[n_{t} /\left(n_{t}+1\right)\right]^{a^{\dagger} a},
$$

is a thermal state with $n_{t}$ average number of photons, the quantity $n_{s}=\sinh ^{2}|r|$ will be referred to as the number of squeezed photons. 
By choosing a suitable rotating frame, the lossless BS exchange interaction is described by the unitary evolution $U_{\tau}=\exp \left[\theta\left(\hat{a}_{1}^{\dagger} \hat{a}_{2}-\hat{a}_{1} \hat{a}_{2}^{\dagger}\right)\right]$, with $\theta \in \mathbb{R}$ and where $\tau=\cos ^{2} \theta$ denotes the transmissivity of the BS. If $\tau=1 / 2$, the BS is said to be balanced. Being a bilinear interaction of modes, this evolution preserves the Gaussian character of the state, and in turn induces a symplectic transformation $S_{\tau}$ in the quantum phase space of the composite system, namely

$$
\mathrm{S}_{\tau}=\left(\begin{array}{cc}
\sqrt{\tau} \mathbb{I} & \sqrt{1-\tau} \mathbb{I} \\
-\sqrt{1-\tau} \mathbb{I} & \sqrt{\tau} \mathbb{I}
\end{array}\right),
$$

where $\mathbb{I}=\operatorname{diag}(1,1)$. Given two uncorrelated singlemode GSs, with CMs $\boldsymbol{\sigma}_{1}$ and $\boldsymbol{\sigma}_{2}$, respectively, the symplectic transformation $S_{\tau}$, acting by congruence on the initial CM $\boldsymbol{\Sigma}_{\mathbf{0}}=\boldsymbol{\sigma}_{1} \oplus \boldsymbol{\sigma}_{2}$, leads to the evolved CM $\boldsymbol{\Sigma}=\mathrm{S}_{\tau} \boldsymbol{\Sigma}_{\mathbf{0}} \mathrm{S}_{\tau}^{T}$. As mentioned above and schematically depicted in Fig. 1, in this work we will consider a bipartite quantum system of modes $\hat{a}_{1}$ and $\hat{a}_{2}$. The mode $\hat{a}_{1}$ is initially in the zero-mean GS $\varrho=\varrho\left(n_{s}, n_{t}\right)$ while mode $\hat{a}_{2}$ is in a thermal state $\nu=\nu\left(n_{2}\right)$. Without loss of generality we will assume a real squeezing parameter $r \in \mathbb{R}$. Using this parametrization, the average number of photons in the first mode $\left\langle\hat{a}_{1}^{\dagger} \hat{a}_{1}\right\rangle_{\varrho} \equiv \operatorname{Tr}\left[\hat{a}_{1}^{\dagger} \hat{a}_{1} \varrho\left(n_{s}, n_{t}\right)\right]$ explicitly reads:

$$
\left\langle\hat{a}_{1}^{\dagger} \hat{a}_{1}\right\rangle_{\varrho}=n_{t}+\left(1+2 n_{t}\right) n_{s} \equiv n_{1} .
$$

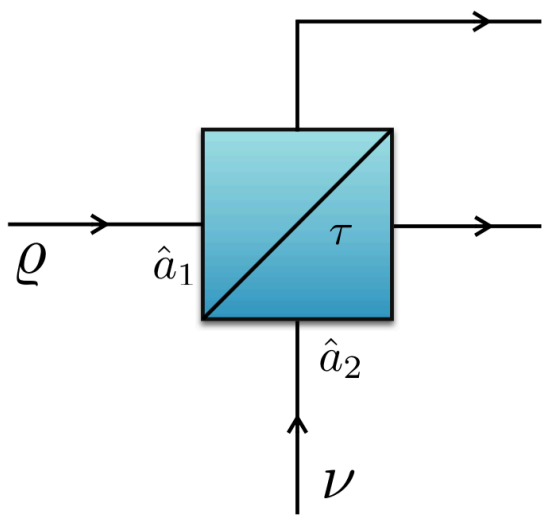

FIG. 1: (Color online) Linear mixing of Gaussian states. The two input modes $\hat{a}_{1}$ and $\hat{a}_{2}$, initially excited in the zero-mean Gaussian state $\varrho=\varrho\left(n_{s}, n_{t}\right)$ and in the thermal state $\nu=$ $\nu\left(n_{2}\right)$ respectively, enter a beam splitter of transmissivity $\tau$, after which quantum correlations are eventually established.

In the phase space, the GSs $\varrho$ and $\nu$ are represented by the $2 \times 2 \mathrm{CMs}$ :

$$
\boldsymbol{\sigma}_{\varrho}=\operatorname{diag}\left(\frac{1}{2}+n_{1}+\Delta, \frac{1}{2}+n_{1}-\Delta\right),
$$

and

$$
\boldsymbol{\sigma}_{\nu}=\left(\frac{1}{2}+n_{2}\right) \mathbb{I}
$$

respectively, where $\Delta=\left(1+2 n_{t}\right) \sqrt{n_{s}\left(1+n_{s}\right)}$. Since the initial state $R_{0}$ of the bipartite system is chosen to be factorized, namely $R_{0}=\varrho \otimes \nu$, the total number of excitations is given by $\left\langle\hat{a}_{1}^{\dagger} \hat{a}_{1}+\hat{a}_{2}^{\dagger} \hat{a}_{2}\right\rangle_{R_{0}}=n_{1}+n_{2} \equiv N$. Now we let the state $R_{0}$ evolve through a lossless BS of transmissivity $\tau$ using the symplectic transformation $\mathrm{S}_{\tau}$ given by Eq. (2). The $4 \times 4 \mathrm{CM}$ of the two-mode output state $R=U_{\tau} R_{0} U_{\tau}^{\dagger}$ is $\boldsymbol{\Sigma}=\mathrm{S}_{\tau}\left(\boldsymbol{\sigma}_{\varrho} \oplus \boldsymbol{\sigma}_{\nu}\right) \mathrm{S}_{\tau}^{T}$ and it reads:

$$
\boldsymbol{\Sigma}=\left(\begin{array}{cccc}
a_{+} & 0 & c_{+} & 0 \\
0 & a_{-} & 0 & c_{-} \\
c_{+} & 0 & b_{+} & 0 \\
0 & c_{-} & 0 & b_{-}
\end{array}\right)
$$

where:

$$
\begin{aligned}
& a_{ \pm}=\left(\frac{1}{2}+n_{2}\right)(1-\tau)+\left(\frac{1}{2}+n_{1} \pm \Delta\right) \tau, \\
& b_{ \pm}=\left(\frac{1}{2}+n_{2}\right) \tau+\left(\frac{1}{2}+n_{1} \pm \Delta\right)(1-\tau), \\
& c_{ \pm}=\left[\left(\frac{1}{2}+n_{2}\right)-\left(\frac{1}{2}+n_{1} \pm \Delta\right)\right] \sqrt{\tau(1-\tau)} .
\end{aligned}
$$

It is useful to introduce the following local symplectic invariants, which in this case are given by $I_{1}=a_{+} a_{-}, I_{2}=$ $b_{+} b_{-}, I_{3}=c_{+} c_{-}$and $I_{4}=\operatorname{det} \boldsymbol{\Sigma}$. Via symplectic diagonalization $\boldsymbol{\sigma}_{A B}$ can be cast into the diagonal form $\operatorname{diag}\left(\lambda_{+}, \lambda_{+}, \lambda_{-}, \lambda_{-}\right)$where the expression of the symplectic eigenvalues is given by [22]:

$$
\lambda_{ \pm}=\sqrt{\frac{I_{1}+I_{2}+2 I_{3} \pm \sqrt{\left(I_{1}+I_{2}+2 I_{3}\right)^{2}-4 I_{4}}}{2}} .
$$

Positivity of $\varrho_{A B}$ requires $\lambda_{-} \geq 1 / 2$.

\section{NONCLASSICALITY FOR BOSONIC SYSTEMS}

Let us now review in some details the concepts of nonclassicality we are going to consider, together with their respective figures of merit. The reader familiar with those concepts can skip this Section.

\section{A. Nonclassicality in the phase space: P-classicality}

Any bipartite bosonic state described by the density matrix $\varrho_{A B}$ can be always expanded in terms of coherent states as follows:

$$
\varrho_{A B}=\int_{\mathbb{C}} d^{2} \alpha \int_{\mathbb{C}} d^{2} \beta P(\alpha, \beta)|\alpha\rangle\langle\alpha|\otimes| \beta\rangle\langle\beta|,
$$

where $|\alpha\rangle$ and $|\beta\rangle$ are coherent states of the two modes and $P(\alpha, \beta)$ is the Glauber-Sudarshan $P$-representation of the state. $P(\alpha, \beta)$ provides a complete characterization of the state. Equation (9) suggests that the state 
of the electromagnetic field can be regarded as a mixture of coherent states, weighted by $P(\alpha, \beta)$. However, in general the $P$-function cannot be regarded as a probability density function. On the other hand, when all the conditions for the $P$-function to be a probability density are satisfied, one can conclude that it describes a classical state of the bosonic field, motivating the following definition:

Definition 1 (P-classicality) A state $\varrho_{A B}$ of a two-mode bosonic field is called $P$-classical if $P(\alpha, \beta)$ is a regular and normalized positive function.

In the case of a single-mode state $\varrho$, we can introduce a generalized $s$-ordered Wigner function which encompasses all the quasi-probability distributions :

$$
W_{s}(\alpha)=\int_{\mathbb{C}} \frac{d^{2} \lambda}{\pi^{2}} e^{\alpha \lambda^{*}-\alpha^{*} \lambda+(s / 2)|\lambda|^{2}} \operatorname{Tr}[D(\lambda) \varrho],
$$

where $D(\alpha) \equiv \exp \left(\alpha \hat{a}^{\dagger}-\alpha^{*} \hat{a}\right)$ is the displacement operator. In the case of $s=-1,0,1$ one recovers the Husimi, Wigner, and Glauber-Sudarshan functions, respectively. The latter, more than any other representation, can depart from being a well-behaved probability density. In order to understand this fact, let us observe that the $s$-ordered Wigner function of a state is related to the $P$ function $(s=1)$ of the same state through a Gaussian convolution, namely:

$$
W_{s}(\alpha)=\frac{2}{\pi(1-s)} \int_{\mathbb{C}} d^{2} \beta \exp \left\{-\frac{2|\alpha-\beta|^{2}}{1-s}\right\} P(\beta),
$$

that can be seen as a smoothing operation. Given the $P$-function of the state of interest, as the parameter $s$ moves towards -1 , the resulting distributions $W_{s}(\alpha)$ get smoother and smoother. Since for $s=-1$ the Husimi $Q$-function is recovered, we obtain a continuous interpolation between $P$ - and $Q$-function, and we are guaranteed that this smoothing operation always succeeds in giving a true probability distribution.

Based on this, it is possible to define a quantitative measure of $P$-nonclassicality, that is the non-classical depth of a quantum state $[23,24]$. To this aim it is useful to introduce the parameter $\mathrm{T}=(1-s) / 2$. For T large enough, Eq. (11) leads to a $P$-classical state and the smoothing operation is referred to as complete. If $\Omega$ denotes the set of all $\mathrm{T}$ which give a complete smoothing of the initial the $P$-function, the non-classical depth is defined as

$$
\mathrm{T}_{m}=\inf _{\mathrm{T} \in \Omega}(\mathrm{T}) .
$$

The non-classical depth ranges from 0 for coherent states to 1 for Fock states [25], whereas for a single-mode GS $\varrho$ we have [26]:

$$
\mathrm{T}_{m}=\max \left[\frac{1}{2}\left(1-\frac{e^{-2 r}}{\mu}\right), 0\right],
$$

where $\mu=\operatorname{Tr}\left[\varrho^{2}\right]$ is the purity of the state and $r$ is the squeezing parameter introduced in Eq. (1).

\section{B. Nonclassicality of correlations: C-classicality}

The total amount of correlations between two classical systems $A$ and $B$ is quantified by the mutual information $I(A: B)=H(A)+H(B)-H(A, B)$ where $H(X)=-\sum_{x} p_{X}(x) \log p_{X}(x)$ is the Shannon entropy of the random variable $X$. By exploiting the relation $p_{A B}(a, b)=p_{A}(a \mid b) p_{B}(b)$ one also gets the equivalent expression of the mutual information $I(A: B)=$ $H(A)-H(A \mid B)$ in terms of the conditional entropy $H(A \mid B)=-\sum_{a, b} p_{B}(b) p_{A}(a \mid b) \log p_{A}(a \mid b)$. The first expression of the mutual information has an immediate extension to quantum systems, simply by replacing the Shannon entropy with the Von Neumann entropy $S[\varrho]=-\operatorname{Tr}[\varrho \log \varrho]$, namely $I_{M}\left(\varrho_{A B}\right)=S\left(\varrho_{A}\right)+S\left(\varrho_{B}\right)-$ $S\left(\varrho_{A B}\right)$; if we address GSs, it can be expressed as [22]

$$
I_{M}\left(\varrho_{A B}\right)=f\left(\sqrt{I_{1}}\right)+f\left(\sqrt{I_{2}}\right)-f\left(\lambda_{+}\right)-f\left(\lambda_{-}\right),
$$

where $f(x)=\left(x+\frac{1}{2}\right) \log \left(x+\frac{1}{2}\right)-\left(x-\frac{1}{2}\right) \log \left(x-\frac{1}{2}\right)$. On the other hand, the extension of the second expression to the quantum realm involves a measurement on one of the two parties, say $B$, described by the positive operationvalued measure $(\mathrm{POVM})\left\{\Pi_{k}\right\}, \Pi_{k} \geq 0, \quad \sum_{k} \Pi_{k}=\hat{\mathbb{I}}$. The probability to obtain the outcome $k$ is given, according to the Born rule, by $p_{k}=\operatorname{Tr}\left[\varrho_{A B} \hat{\mathbb{I}} \otimes \Pi_{k}\right]$ and the conditional state of $A$ with respect to the $k$ outcome $\varrho_{A \mid B}^{\Pi_{k}}=\left(p_{k}\right)^{-1} \operatorname{Tr}_{B}\left[\varrho_{A B} \hat{\mathbb{I}} \otimes \Pi_{k}\right]$. The maximum amount of information we can gain on the part $A$ by locally measuring the other part thus has the non-trivial expression

$$
\mathcal{C}_{A \mid B}\left(\varrho_{A B}\right)=\max _{\left\{\Pi_{k}\right\}}\left\{S\left(\varrho_{A}\right)-\sum_{k} p_{k} S\left(\varrho_{A \mid B}^{\Pi_{k}}\right)\right\},
$$

and invokes an optimization procedure over the set of all measurements. The quantum discord is properly defined as the difference between these two quantities [27]

$$
\mathcal{D}_{A \mid B}\left(\varrho_{A B}\right)=I_{M}\left(\varrho_{A B}\right)-\mathcal{C}_{A \mid B}\left(\varrho_{A B}\right) .
$$

We can thus conclude that a system shows some quantumness as soon as the discord is different from zero, providing us with the following criterion:

Definition 2 (C-classicality) A state $\varrho_{A B}$ of a twomode bosonic field is called $C$-classical if $\mathcal{D}_{A \mid B}\left(\varrho_{A B}\right)=$ $\mathcal{D}_{B \mid A}\left(\varrho_{A B}\right) \equiv 0$.

If we restrict to the subclass of GSs and Gaussian measurements, an analytical expression of the quantum discord can be derived, which is called Gaussian discord, and is given by

$$
\mathcal{D}_{A \mid B}\left(\varrho_{A B}\right)=f\left(\sqrt{E_{A \mid B}^{\min }}\right)+f\left(\sqrt{I_{2}}\right)-f\left(\lambda_{+}\right)-f\left(\lambda_{-}\right),
$$

where $E_{A \mid B}^{\min }$ has an analytical expression as a function of the local symplectic invariants $I_{k}$ [28-32]. It is worth noting that for a large class of Gaussian states [33] the 
Gaussian discord of Eq. (17) coincides with the quantum discord, i.e. the maximum in Eq. (15) is achieved by a Gaussian measurement.

A stronger form of quantum correlations with respect to discord is given by quantum entanglement. In the case of two-mode GSs a necessary and sufficient condition can be derived to assess the presence of entanglement [34]. It is essentially based on the positivity of $\varrho_{A B}$ under partial transposition (PPT), that is the positivity of the density matrix obtained by the transposition applied only to one part of a system [35]. One can show in fact that the symplectic eigenvalues of the partially transposed states are given by:

$$
\tilde{\lambda}_{ \pm}=\sqrt{\frac{I_{1}+I_{2}-2 I_{3} \pm \sqrt{\left(I_{1}+I_{2}-2 I_{3}\right)^{2}-4 I_{4}}}{2}},
$$

and a Gaussian state $\varrho_{A B}$ is entangled if and only if $\tilde{\lambda}_{-}<1 / 2$. In fact, a measure of entanglement is given by the logarithmic negativity [36], that is $E(\boldsymbol{\sigma})=$ $\max \left[-\log \left(2 \tilde{\lambda}_{-}\right), 0\right]$.

\section{NON-CLASSICALITY ARISING FROM MIXING A GAUSSIAN STATE WITH THE VACUUM}

We start considering the case in which the reference input state of mode $\hat{a}_{2}$ is the vacuum, i.e. $n_{2}=0$ and, in particular $\boldsymbol{\sigma}_{\nu} \rightarrow \boldsymbol{\sigma}_{0} \equiv \frac{1}{2} \mathbb{I}$ and $n_{1}=N$.

\section{A. P-classicality}

The initial state of the system $R_{0}$ is clearly $C$-classical. On the contrary, $P$-nonclassicality has to be addressed both in the input and output channels, in order to see wether differences arise. The Glauber-Sudarshan $P$ representation of $R_{0}$ is given by

$$
R_{0}=\int_{\mathbb{C}} d^{2} \alpha \int_{\mathbb{C}} d^{2} \beta P_{\varrho}(\alpha) P_{0}(\beta)|\alpha\rangle\langle\alpha|\otimes| \beta\rangle\langle\beta|,
$$

where the $P$-function $P_{R_{0}}$ is factorized in the product of $P_{\varrho}$ and $P_{0}$, being the latter the Glauber-Sudarshan $\mathrm{P}$-function of the vacuum. Moreover, since $P_{0}$ is a wellbehaved probability density, any possible $P$-nonclassical feature of the input state is due to the pathological behavior of $P_{\varrho}$ alone, and is quantified by the nonclassical depth of Eq. (12). Since the action of the BS evolution on the two-mode displacement operator is $U_{\tau} D_{a}(\alpha) \otimes$ $D_{b}(\beta) U_{\tau}^{\dagger}=D_{a}(\sqrt{\tau} \alpha+\sqrt{1-\tau} \beta) D_{b}(\sqrt{\tau} \beta-\sqrt{1-\tau} \alpha)$, and it amounts to a rotation of the arguments, one obtains the following $P$-representation of $R$ :

$$
P_{R}(\alpha, \beta)=P_{\varrho}(\sqrt{\tau} \alpha-\sqrt{1-\tau} \beta) P_{0}(\sqrt{1-\tau} \alpha+\sqrt{\tau} \beta) .
$$

It is apparent that the effect of the evolution only amounts to a re-parametrization of the argument, that does not affect the functional form of both $P_{\varrho}$ and $P_{0}$. Thus we can conclude that output state $R$ is $P$ nonclassical if and only if $\varrho$ is $P$-nonclassical, that is, for our configuration the two-mode P-nonclassicality of the output equals single-mode $P$-nonclassicality of the input. Let us remark, for the sake of Sec.V, that the foregoing argument applies as well for the case of mixing with a reference thermal state with positive temperature, given that the P-function of a general thermal state is a wellbehaved probability density. The non-classical depth in Eq. (12) relative to the mode $\hat{a}_{1}$, can be expressed as:

$$
\mathrm{T}_{m}=\max \left[\frac{1-2 u}{2}, 0\right]
$$

where $u=\frac{1}{2}+n_{1}-\Delta$ is the minimum eigenvalue of the $\mathrm{CM} \boldsymbol{\sigma}_{\varrho}$, as it is apparent from Eq. (4). The condition $\mathrm{T}_{m}=0$ singles out a $P$-classicality threshold, which can be made explicit either as a function of the thermal component $n_{t}$, hence having

$$
n_{s}^{\mathrm{P}}=\frac{n_{t}^{2}}{1+2 n_{t}},
$$

or of the squeezed ones

$$
n_{t}^{\mathrm{P}}=n_{s}+\sqrt{n_{s}\left(1+n_{s}\right)} .
$$

Whenever the average number of squeezed photons exceeds $n_{s}^{\mathrm{P}}$, or the thermal component falls below $n_{t}^{\mathrm{P}}$ the state $\varrho$ turns out to be $P$-nonclassical. From now on, otherwise differently stated, $n_{s}^{\mathrm{P}}$ shall be employed as the $P$-classicality threshold.

\section{B. C-classicality and generation of Gaussian discord}

We now address general quantum correlations, and investigate the generation of Gaussian discord. Being the parties involved the output modes $\hat{b}_{1}=U^{\dagger} \hat{a}_{1} U$ and $\hat{b}_{2}=U^{\dagger} \hat{a}_{2} U$, we will refer to $\mathcal{D}_{1 \mid 2}$ as the $b_{1}$-discord and $\mathcal{D}_{2 \mid 1}$ to the $b_{2}$-discord; when they both coincide the symbol $\mathcal{D}$ will be employed employed. In fact, this is the case of a balanced BS, namely $\mathcal{D}_{1 \mid 2}\left(n_{s}, n_{t}, 1 / 2\right)=$ $\mathcal{D}_{2 \mid 1}\left(n_{s}, n_{t}, 1 / 2\right) \quad \forall n_{s}, n_{t}$.

In Fig. 3 we show a plot of the Gaussian discord as a function of the squeezed and thermal component of the input state $\varrho$, for the balanced case $\tau=1 / 2$. Except for the trivial case of a vacuum input state $\varrho=|0\rangle\langle 0|$, it is apparent that the discord is always positive and therefore, contrary to the case of P-classicality, there is no $C$-classicality threshold: whatever the input state, a balanced BS is capable of generating quantum correlations. This is also in agreement with the fact that typically almost all states possess positive discord [45]. 


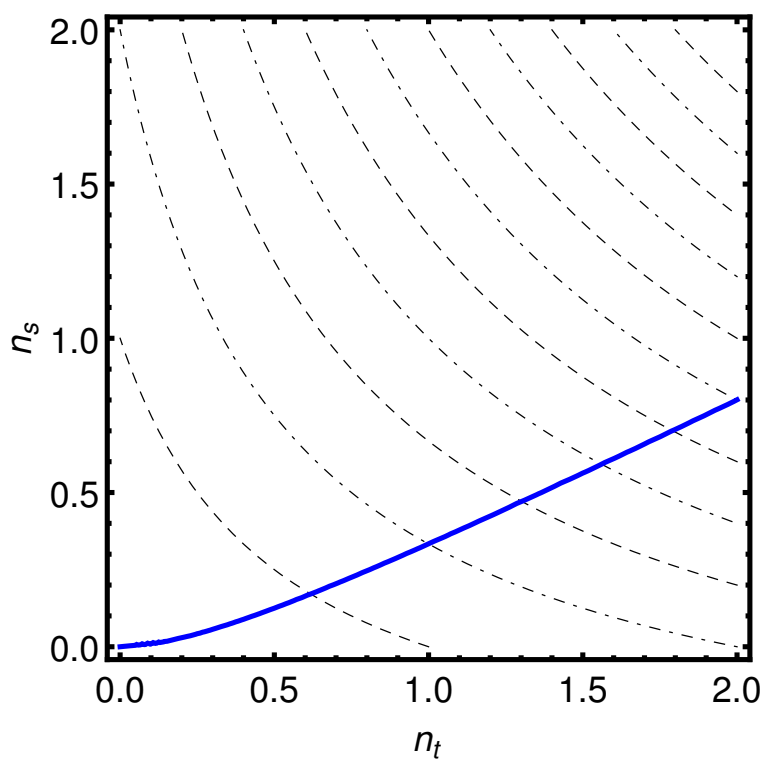

FIG. 2: (Color online) Nonclassicality by mixing with the vacuum: Plot of the implicit function $\mathrm{T}_{m}=0$, which defines the $P$-classicality threshold. It takes the explicit expression of Eq. (22) as a function of $n_{t}$, while as a function of $n_{s}$ it is given by Eq. (23). In this case the non-classicality threshold $n_{s}^{\mathrm{P}}$ and the separability threshold $n_{s}^{\text {sep }}$ coincide. The black lines are curves of fixed total energy $N=n_{s}+n_{t}+2 n_{s} n_{t}$ : dashed for odd values $N=2 k+1, k=0,1, \ldots$ and dot-dashed for even values $N=2 k, k=1,2, \ldots$ of the total energy.

From Fig. 3 a quantitatively relevant feature emerges. Considering input states below the $P$-classicality threshold (denoted by the dashed black curve), one can observe that the output discord and hence the $C$-nonclassicality increases as the input non-classical resources decreases. This is true regardless the constraints that one considers: either moving along the curves at constant $n_{s}, n_{t}$, or total energy $N$, the discord increases as $n_{s}$ decreases or $n_{t}$ increases. This is a quantitative feature that strikingly confirms - together with the absence of a $C$-classicality threshold - the inequivalence between the two notions of classicality considered here.

In Fig. 3 the Gaussian discord corresponding to three families of input states has been highlighted: the discord generated by a thermal input state $\mathcal{D}^{\text {th }}=\mathcal{D}\left(0, n_{t}\right)$ corresponds to the solid blue curve; the dotted red line is obtained when the input state is the squeezed vacuum state, i.e. $\mathcal{D}^{\mathrm{sq}}=\mathcal{D}\left(n_{s}, 0\right)$; and the black dashed line represents the value of the discord at the $P$-classicality threshold, i.e. $\mathcal{D}^{\mathrm{P}}=\mathcal{D}\left(n_{s}^{\mathrm{P}}, n_{t}\right)$. For these limiting cases, analytical expressions of the discord in terms of the total energy $N$ of are available, even if quite cumbersome, and hence not reported. Being functions of a single quantity, they are suitable for comparison and have been plotted in the lower panel of Fig. 3, together with the relative values of the classical correlations $C=I_{M}-\mathcal{D}$. Moreover, in Fig. 3 we also show, by a dashed magenta line, the curve corresponding to the minimum value attained
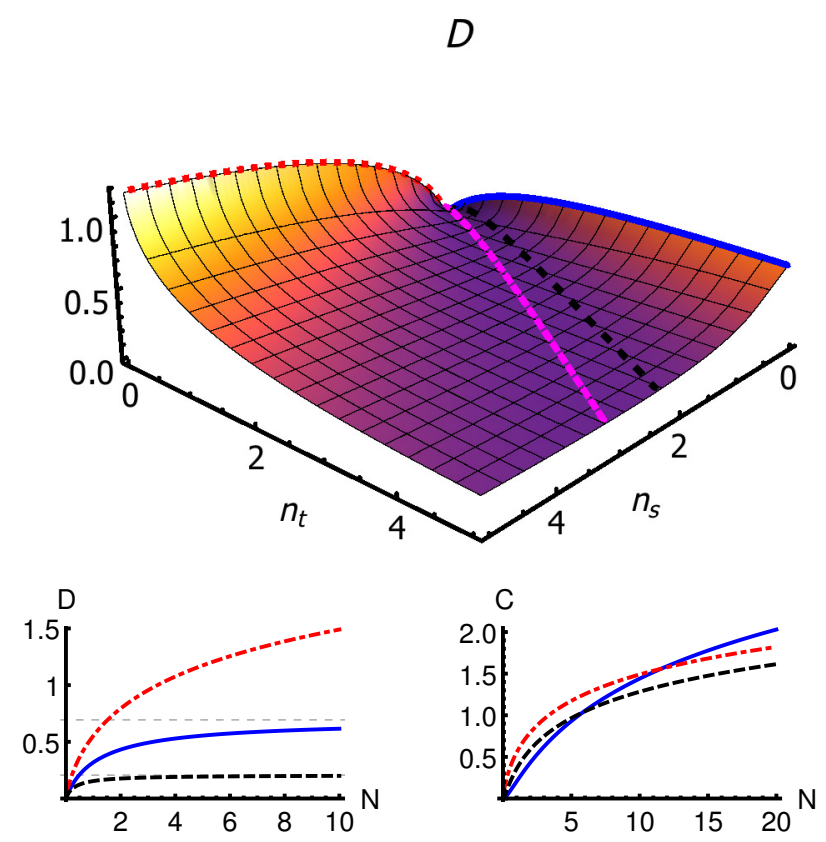

FIG. 3: (Color online) Nonclassicality by mixing with the vacuum: In the upper panel we show the discord $\mathcal{D}$ as a function of the number of squeezed and thermal photons $n_{s}, n_{t}$ for a balanced BS $\tau=1 / 2$. The dotted red line corresponds to the case of a squeezed vacuum state entering the beam splitter. The solid blue line corresponds to a thermal input state, while the dashed black curve is the discord at the $P$-classicality threshold. Finally the dashed magenta curve points out the minimum value of the discord, obtained via numerical maximization. The lower left panel shows the discord as a function of the total energy $N$; solid blue for thermal state, dashed red for squeezed vacuum, black dot-dashed for $P$-classicality threshold. The right panel shows the classical correlations for the same input states and with the same color codes.

by the discord (for fixed $n_{t}$ ) obtained via numerical minimization.

From the left bottom panel in Fig. 3, we can see that the discord is a monotonically increasing function of the total energy $N$. The discord saturates to a finite value both for a thermal input state, for which we find

$$
\lim _{N \rightarrow \infty} \mathcal{D}^{\text {th }}=\log 2,
$$

and at the $P$-classicality threshold [46], where

$$
\lim _{N \rightarrow \infty} \mathcal{D}^{\mathrm{P}}=\frac{1}{2} \log (3+2 \sqrt{2})-\frac{3}{2} \log \sqrt{2} \approx 0.2067 .
$$

Again we see that, for a fixed value of the total energy, a thermal input state results in more quantum correlations than a state lying on the non-separability boundary, although in the latter case squeezing is involved. Actually, as we can see from Fig. 3, the states corresponding to the $P$-classicality threshold do not correspond to the states with minimum output discord (dashed magenta curve), confirming again the inequivalence between $P$ - 
and $C$-classicality. The minimum output discord curve has been obtained numerically and we have not found any clear physical picture of the class of states for which this minimum is achieved. On the other hand, for a squeezed vacuum state the discord grows logarithmically for large $N$ values. As a final remark, from the right plot in the lower panel of Fig. 3, it is apparent that as the energy increases the classical correlations always increase indefinitely, whereas as said, below the $P$-classicality threshold discord is bounded. This behavior will become clear in the next section, where entanglement will be considered.

If we now release the restriction of a balanced BS and inquire the behavior of the discord with respect to $\tau$, we see that $\mathcal{D}_{1 \mid 2}(R)$ and $\mathcal{D}_{2 \mid 1}(R)$ differ from each others; for a generic value $\tau$ of the transmissivity, $b_{1}$-discord and $b_{2}$-discord are simply related by an exchange of the symplectic invariants of $I_{1}$ and $I_{2}$, which amounts to a swap of the BS transmissivity from $\tau$ to $1-\tau$. In Fig. 4, the $b_{1}$-discord has been plotted as a function of $\tau$, for the relevant cases already mentioned. Being obtained by the exchange of transmissivity and reflectivity, the $b_{2}$-discord is simply given by a reflection about the axis $\tau=\frac{1}{2}$. Of course, in the limiting cases of transmissivity 0 and 1 , the discord falls to zero. Furthermore, apart for a squeezed vacuum input state, the behavior of $\mathcal{D}_{1 \mid 2}(R)$ is not symmetric with respect to $\tau=\frac{1}{2}$. By increasing the incoming energy, the maximum of $\mathcal{D}_{1 \mid 2}(R)\left(\mathcal{D}_{2 \mid 1}(R)\right)$, and hence the optima transmissivity, shifts towards $\tau=1(0)$.

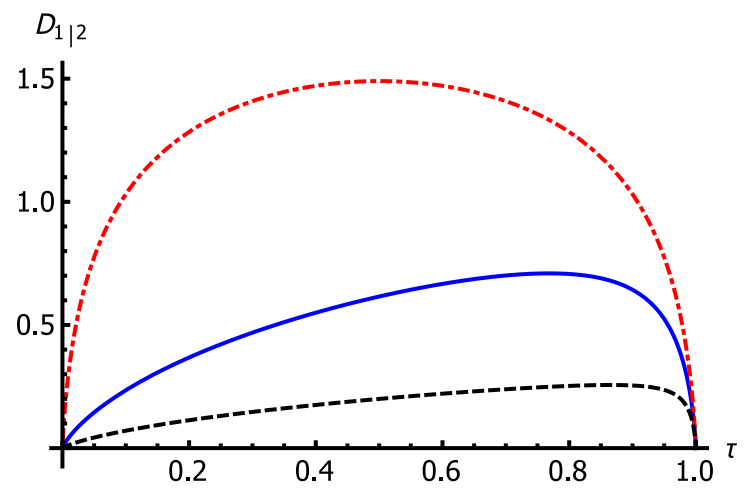

FIG. 4: (Color online) Nonclassicality by mixing with the vacuum: plot of the discord $\mathcal{D}_{1 \mid 2}(R)$ as a function of the transmissivity $\tau$ for a given total energy $N=10$. Colors as in Fig. 3 .

\section{Generation of Gaussian entanglement}

Let us now focus on the generation of Gaussian entanglement. The explicit expressions of the symplectic invariants are given by

$$
\begin{aligned}
& I_{1}=\frac{1}{4}+n_{t}\left(1+n_{t}\right) \tau+N(1-\tau) \tau, \\
& I_{2}=\frac{1}{4}+n_{t}\left(1+n_{t}\right)(1-\tau)+N(1-\tau) \tau, \\
& I_{3}=-\left[\left(1-n_{t}\right) n_{t}+N\right](1-\tau) \tau, \\
& I_{4}=\frac{1}{16}\left(1+2 n_{t}\right)^{2} .
\end{aligned}
$$

By solving the equation $\tilde{\lambda}_{-}\left(n_{s}, n_{t}, \tau\right)=\frac{1}{2}$ with respect to $n_{s}$, one finds an analytic expression for the the number of squeezing photons at the separability threshold:

$$
n_{s}^{\text {sep }}=\frac{n_{t}^{2}}{1+2 n_{t}},
$$

which does not depend on $\tau$ and, most important, it equals the $P$-classicality threshold Eq. (22). This is in agreement with the general fact that $P$-nonclassicality is necessary and sufficient for the generation of entanglement at a BS, regardless the Gaussian nature of the input state [37-44].

We are now going to analyze more in details the relationship between the generation of Gaussian discord and entanglement. Since, although analytical, the expression of the Gaussian discord is far too involved, being in particular non invertible, we proceed in our analysis by randomly sampling a large number of input Gaussian states and making them evolve trough a BS of random transmissivity $\tau \in[0,1]$; for each of them, minimum symplectic eigenvalue of the partially transposed CM and Gaussian discord are then computed.

The results are shown in Fig. 5 (light gray points), together with the plot obtained for evolutions trough a balanced BS (dark gray points). Inspecting the latter distribution it is easy to recover all the features already addressed in Fig. 3. In particular, since for input thermal states of large energy the discord has been found to reach the limiting value $\log 2 \approx 0.6931$, the distribution displays an asymptote, so that we can conclude that the region of high $\tilde{\lambda}$ corresponds to highly excited input thermal states. Moreover, as it can be seen following the dashed black line in Fig. 3, by moving on the separability threshold, i.e. considering the points laying on the line $\tilde{\lambda}_{-}=1 / 2$, we move from zero discord to the asymptotic value of Eq. (24), which is obtained for infinite input energy and pointed out by the black arrow. It is also possible to note that the minimum value of the discord is attained slightly below $\tilde{\lambda}_{-}=1 / 2$, as shown by the dashed magenta line of Fig. 3 .

More in general, considering arbitrary transmissivity (light gray points in Fig. 5) if the evolved state has a discord $\mathcal{D}_{1 \mid 2}(R)>1$, it will be necessarily entangled $[28,29]$ : the avoided region of the plane $\left\{\left(\mathcal{D}, \tilde{\lambda}_{-}\right) \mid \mathcal{D}>1, \tilde{\lambda}_{-}>\right.$ $1 / 2\}$ shows that the discord for separable states is always smaller than one. It means that for separableand hence $P$-classical - input states, whatever the transmissivity, the discord between the output modes cannot 


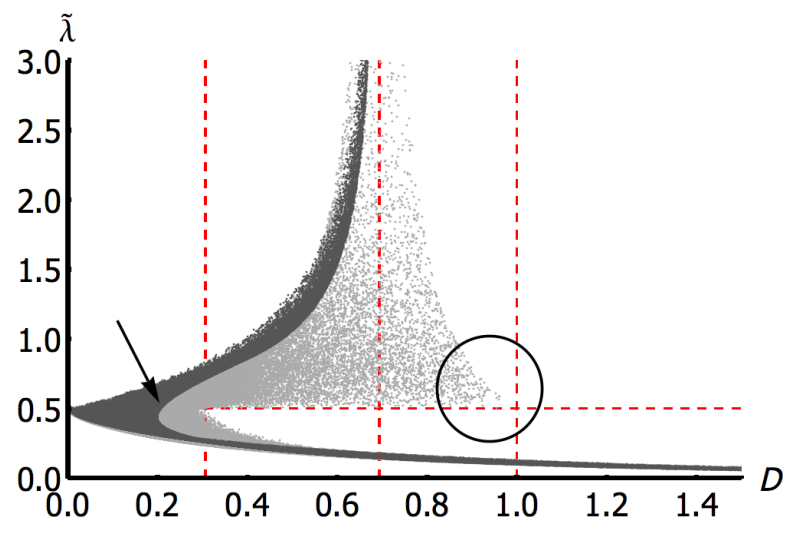

FIG. 5: (Color online) Nonclassicality by mixing with the vacuum: plot of the minimum symplectic eigenvalue of the partial transpose CM $\tilde{\lambda}_{-}$versus the discord $\mathcal{D}_{1 \mid 2}(R)$ for randomly generated input states $\varrho\left(n_{s}, n_{t}\right)$ evolving through a balanced BS (dark gray points), and for random values of the transmissivity $\tau$ (light gray points). The vertical dashed lines correspond to $1-\log 2 \approx 0.3069, \log 2 \approx 0.6931$, which is an asymptotic value for thermal states entering a balance BS, and 1 , beyond which only entangled states $\left(\tilde{\lambda}_{-}<1 / 2\right)$ can be found. The black circle stresses the portion of the plane occupied by states attaining the maximum value of the discord, still being separable; the black arrow points the maximum value of the discord achieved at the separability threshold $\tilde{\lambda}_{-}$ $=1 / 2$ in the case of a balanced BS.

grow indefinitely [29], by simply pumping more energy. On the other hand, in the region $0 \leq \mathcal{D}_{1 \mid 2}(R) \leq 1$, both entangled and separable states are present. Another region of interest is the entangled region, namely $\left\{\left(\mathcal{D}, \tilde{\lambda}_{-}\right) \mid \tilde{\lambda}_{-} \leq 1 / 2\right\}$, where the random generated points get "horn-shaped". One remarkable feature is that, when $\tilde{\lambda}_{-}$approaches zero, i.e. entanglement is high, the discord becomes, loosely speaking, nearly a function of $\tilde{\lambda}_{-}$, and hence of entanglement itself. In this case, we note that for $\mathcal{D} \gtrsim 1$ the extent of the region in Fig. 5 is bounded by two convergent quantities. To better clarify this point we set $\tau=1 / 2$ and consider an input squeezed vacuum state. For large $N \gg 1$ the analytic expressions of the discord $\mathcal{D}^{\text {sq }}$ reads (at the leading order):

$$
\mathcal{D}^{\mathrm{sq}} \approx \log \left(\frac{\sqrt{N}}{2}\right)+1,
$$

while the minimum symplectic eigenvalue of the partial transpose $\tilde{\lambda}_{-}^{\mathrm{sq}}$ is

$$
\tilde{\lambda}_{-}^{\mathrm{sq}} \approx \frac{1}{4 \sqrt{N}}
$$

respectively. Form the previous equations it follows that in this limit $\tilde{\lambda}_{-}^{\mathrm{sq}} \approx e^{1-\mathcal{D}^{\mathrm{sq}}} / 8$, valid for high discord value. It turns out to be an upper bound for the random distribution of symplectic eigenvalues in the entangled region, and hence will be denoted as $\tilde{\lambda}_{-}^{M}$. Upon omitting the superscript sq, we may write

$$
\tilde{\lambda}_{-} \leq \tilde{\lambda}_{-}^{M} \approx \frac{e^{1-\mathcal{D}}}{8} \quad \text { for } \quad \mathcal{D} \gtrsim 1
$$

Analogously, our numerical analysis in the same region shows that the random generated points are always bounded from below by $\tilde{\lambda}_{-}^{m}$, whose expression is

$$
\tilde{\lambda}_{-} \geq \tilde{\lambda}_{-}^{m} \approx \frac{e^{-\mathcal{D}}}{4} \quad \text { for } \quad \mathcal{D} \gtrsim 1
$$

Putting together Eqs. (29) and (30) we conclude that, for a fixed value of the discord $\mathcal{D} \gtrsim 1$, the distribution of minimum symplectic eigenvalues of the partially transpose $\mathrm{CM}$, is constrained in the range

$$
\tilde{\lambda}_{-}^{m} \leq \tilde{\lambda}_{-} \leq \tilde{\lambda}_{-}^{M} .
$$

Therefore, for $\mathcal{D} \gg 1, \tilde{\lambda}_{-}$is an exponentially decreasing function of the Gaussian discord.

Particularly interesting is finally the region corresponding to highly discordant - yet separable - states, stressed by a circle in Fig. 5. These are states sharing the maximum amount of quantum correlations without invoking entanglement. We found that these states are obtained for high input energies, whose value can also be due uniquely to thermal photons, entering a BS of extremely high transmissivity, namely $\tau=1-\varepsilon$. Having unlimited thermal resources at disposal, we can still generate quantum correlated output states up to a value $\mathcal{D}_{1 \mid 2}=1$, by sending a very excited input state in an unbalanced BS of very high transmissivity. If, always keeping the BS transmissivity close to one, the fraction of squeezed photons is such to render the input state $P$ nonclassical one, the corresponding points in the plane will lie just below the separability threshold, but the value of the discord can increase only up to the value $1-\log 2 \approx 0.3069$ (indicated by a red dashed line). By further increasing the amount of squeezing, the resulting states will eventually occupy more entangled and more discordant regions of the lower branch.

\section{NONCLASSICALITY ARISING FROM MIXING A GAUSSIAN STATE WITH A THERMAL STATE}

We now consider the general case, allowing for thermal photons to enter the second port of the BS. In fact, in practical scenarios a certain amount of thermal noise (e.g., in the form of black body radiation or scattered light) unavoidably participates in the interference phenomenon and affects the statistics of the outgoing fields.

Though $P$-classicality, as already discussed, retains the expression (22) for the threshold value, the presence of another source of photons affects the properties of the output state. The relevant changes both to quantum 

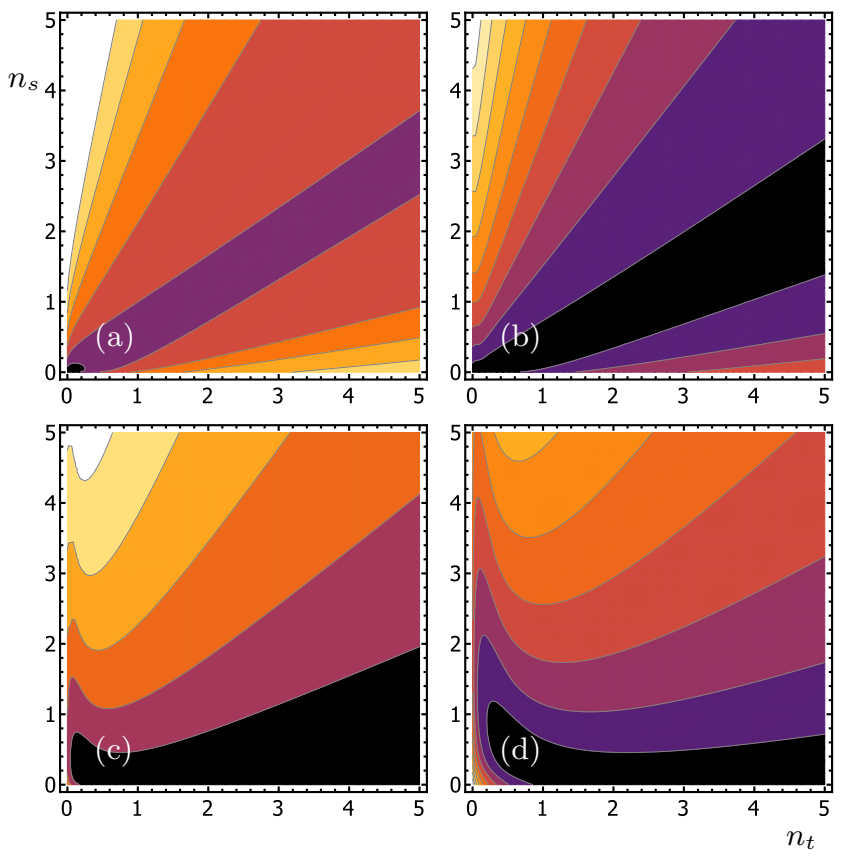

FIG. 6: (Color online) Nonclassicality by mixing with a thermal state: contour plots of Gaussian discord as a function of squeezed and thermal photons $n_{s}$ and $n_{t}$ in mode $a_{1}$. From panel (a) to (d) the number thermal photons in mode $a_{2}$ are given by $n_{2}=0,0.1,1,3$ [panel (a) is in fact the contour plot of Fig. 3]. Colors go from black (0.) to white (0.7).

discord and entanglement can be again evaluated via the symplectic invariants, which now reads

$$
\begin{aligned}
I_{1}= & \frac{1}{4}+n_{2}^{2}(1-\tau)^{2}+\tau\left[n_{t}+n_{s}\left(1+2 n_{t}\right)(1-\tau)+\tau n_{t}^{2}\right] \\
& +n_{2}(1-\tau)\left[1+2 n_{1} \tau\right]
\end{aligned}
$$$$
I_{2}=\frac{1}{4}+n_{t}^{2}(1-\tau)^{2}+\tau\left[n_{2}+n_{s}\left(1+2 n_{2}\right)(1-\tau)+\tau n_{2}^{2}\right]
$$$$
+n_{t}(1-\tau)\left[1+2\left(n_{s}+n_{2}+2 n_{s} n_{2}\right) \tau\right]
$$$$
I_{3}=\left[n_{2}^{2}+n_{t}^{2}-n_{s}\left(1+2 n_{t}\right)-2 n_{1} n_{2}\right](1-\tau) \tau,
$$$$
I_{4}=\frac{1}{16}\left(1+2 n_{t}\right)^{2}\left(1+2 n_{2}\right)^{2},
$$

whence, recalling the expression for the total energy in mode $\hat{a}_{1}$ Eq. (3), we can see that $I_{1}$ and $I_{2}$ are each other related via the exchange of the number of thermal photons $n_{t}$ and $n_{2}$.

\section{A. Generation of Gaussian discord}

As in the previous Section, we first focus on the case of a balanced BS $(\tau=1 / 2)$. In Fig. 6 we show the contours of quantum discord as a function of $n_{s}$ and $n_{t}$ for different thermal-photon number $n_{2}$ of mode $a_{2}$. We can see that, despite the $P$-classicality of the output state remains invariant, the $C$-classicality is much affected by the presence of an additional source of thermal photons. In particular, for a low number of thermal photons $n_{2}$ the region with minimal discord (darker areas in Fig. 6) localizes close to the $P$-classicality threshold, whereas it tends to get closer to the zero squeezed-photon axis for larger $n_{2}$. Also in this case, the inequivalence of the two notions of non-classicality is apparent.

Since there is no threshold for the production of discord, it is legitimate to enquire the generation of quantum correlations at a BS for the "cheapest" conceivable scenario, namely having at disposal only thermal resources in input. Given a certain amount of total thermal photons $N=n_{1}+n_{2}$, which is the most convenient redistribution of the total energy between the two modes, in order to maximize the Gaussian discord at the output? The answer to this question is shown in In Fig. 7, where $b_{1}$ discord as a function of the photon imbalance $d=n_{1}-n_{2}$ between the two input modes has been plotted. For each transmissivity, the $b_{1}$-discord is a monotonically increasing function of the imbalance $d$, so we can conclude that the optimal configuration is the most asymmetric one, where all the thermal photons are sent in on channel, leaving the other in the vacuum state. Moreover, an even distribution of photons $(d=0)$ between the input modes always leads to zero output discord. This fact is apparent by looking at Eq. (7) where for equal input states, no matter the transmissivity, the correlation terms $c_{ \pm}$of the CM identically vanish: the phenomenon is referred to as transparency, since the evolution trough the BS does not leave any imprint on the input states. Being the optimal configuration the one with a thermal input in one port of the BS and the vacuum in the other, we already know that, for a given amount of energy $N$, there will be an optimal value of the transmissivity maximizing the $b_{1}$ discord (as shown by the blue curve of Fig. 4); this fact is also manifest in the crossing of the blue curve and the red dot-dashed one in Fig. 7, corresponding to $\tau=0.5$ and $\tau=0.8$ respectively, when approaching the maximum imbalance. Finally, the dashed curve corresponds to an extremely unbalanced BS, namely $\tau=0.99$, and, provided that high-enough thermal energy is available, the corresponding value of the $b_{1}$-discord close to the imbalance would be the greatest, eventually achieving the limiting value of $\mathcal{D}_{1 \mid 2}=1$, as discussed above for the circled points in Fig. 5 .

\section{B. Generation of Gaussian entanglement}

As before, the equation $\tilde{\lambda}\left(n_{s}, n_{t}, n_{2}, \tau\right)=1 / 2$, if solved with respect to the squeezed number of photon $n_{s}$, gives a threshold on the generation of entanglement when $n_{2}$ thermal photons enter the BS. The explicit expression of 


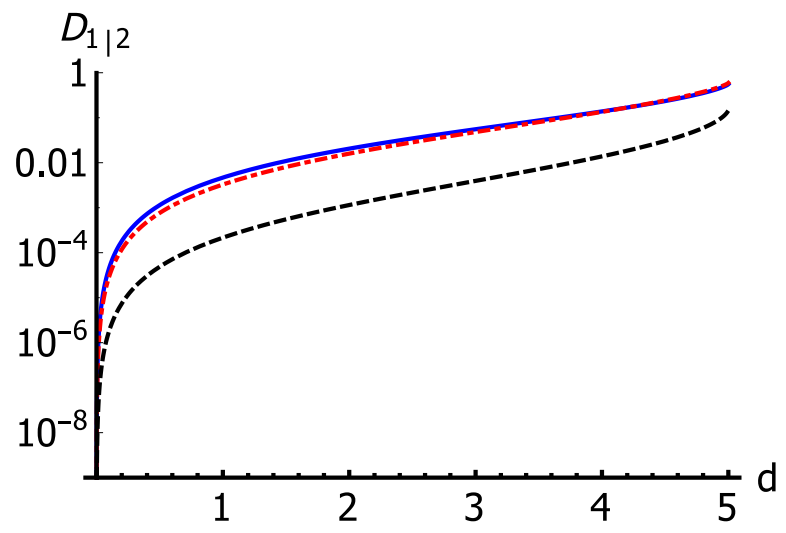

FIG. 7: (Color online) Nonclassicality by mixing with a thermal state: logarithmic plot of the $b_{1}$-discord $\mathcal{D}_{1 \mid 2}(R)$ as a function of the imbalance $d$ for different values of the transmissivity $\tau$ and fixed total energy $N=5$. The solid blue curve is for a balanced $\mathrm{BS} \tau=0.5$, the dot-dashed red line is for $\tau=0.8$ while the black dashed line is for $\tau=0.99$.

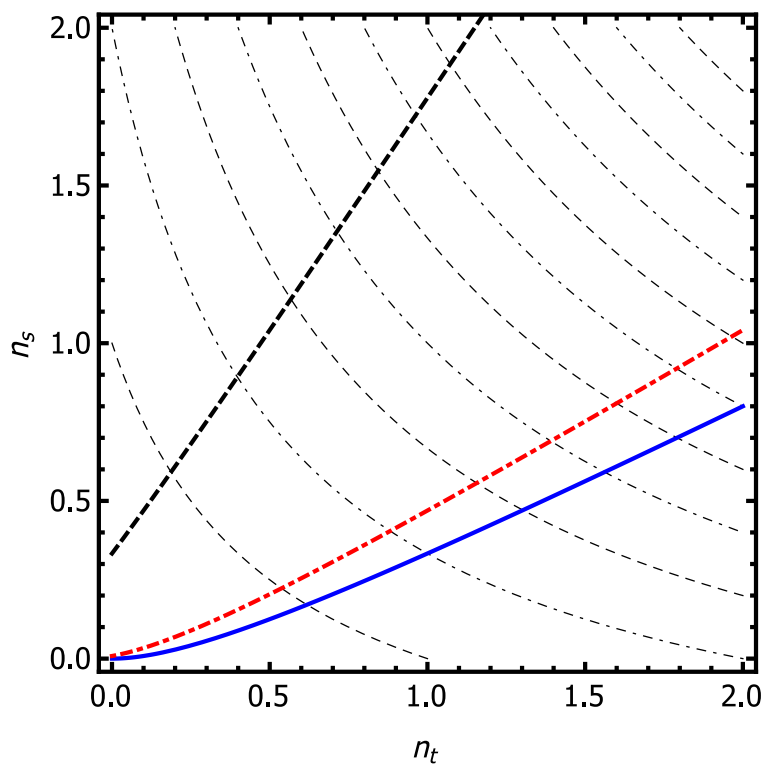

FIG. 8: (Color online) Nonclassicality by mixing with a thermal state: plot of the separability thresholds $n_{s}^{\text {sep }}$ for fixed $\tau=\frac{1}{2}$ and different values of $n_{2}$. The solid blue line corresponds to $n_{2}=0$, and indeed coincides with the nonclassicality threshold $n_{s}^{\text {nc }}$. The red dot-dashed lines represents $n_{s}^{\text {sep }}$ for $n_{2}=0.1$, and finally the black dashed one is for $n_{2}=1$. The black lines are curves of fixed energy in the mode $\hat{a}_{1} n_{1}=n_{s}+n_{t}+2 n_{s} n_{t}$ : dashed for odd values $n_{1}=2 k+1, k=0,1, \ldots$ and dot-dashed for even values $n_{1}=2 k, k=1,2, \ldots$ of the total energy.

$n_{s}^{\text {sep }}$ is given by

$$
n_{s}^{\text {sep }}=\frac{\mu_{1} \mu_{2}}{\tau(1-\tau)} \Theta_{t, 2} \Theta_{2, t}
$$

where $\Theta_{k, l}=n_{k} n_{l}+n_{k}-\left(n_{k}-n_{l}\right) \tau$ and $\mu_{1,2}$ are the purities of the two input states.
Contrary to the vacuum case, it is apparent that in the presence of a thermal state the separability threshold $n_{s}^{\text {sep }}$ and the non-classicality threshold $n_{s}^{\mathrm{nc}}$ are no longer coincident. In Fig. 8 several separability thresholds are shown, for different values of $n_{2}$. As soon as $n_{2}$ differs from zero, the number of squeezed photons $n_{s}$ required to have entanglement increases - as shown both in Fig. 8 and Fig. 9. The previous symmetry between

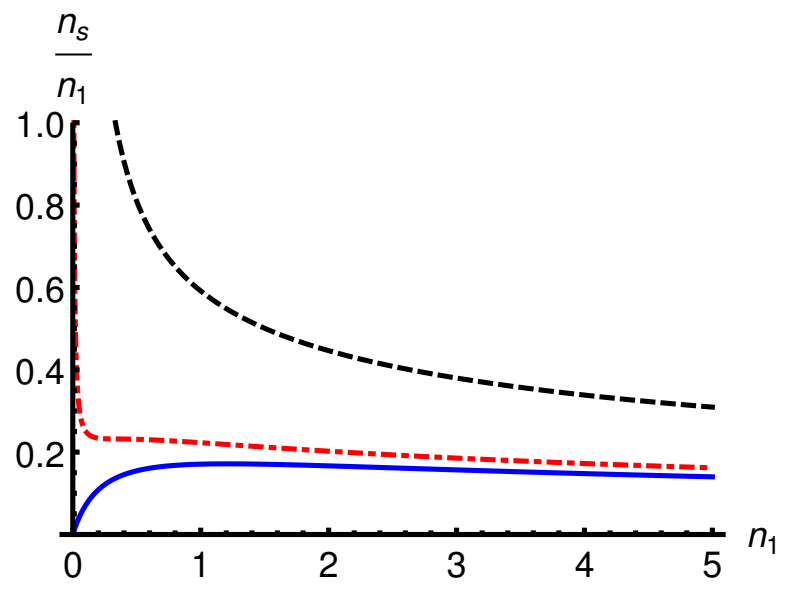

FIG. 9: (Color online) Nonclassicality by mixing with a thermal state: plot of the squeezed fraction of photons $n_{s} / n_{1}$ at the separability threshold, as a function of the total number of photons $n_{1}=n_{s}+n_{t}+2 n_{s} n_{t}$ entering the first port of the beam splitter, for different values of $n_{2}$. The solid blue line corresponds to $n_{2}=0$, The red dot-dashed line corresponds to $n_{2}=0.1$, while the black dashed one to $n_{2}=1$.

the notions of non-classicality in the phase space and non-separability no longer holds: there exists $P$-singular input states of the electromagnetic field, and hence $P$ singular output states, which nevertheless are not entangled. We can conclude that a hierarchy of non-classicality has settled down: non-separability at the output imposes a stricter notion of quantumness than the one put forward by $P$-singular distributions. Injecting into the BS a non-classical state is no longer a sufficient condition to get entanglement between the output modes.

In order to better investigate this point, we express the separability threshold relative to $n_{2}$ thermal photons as a function of the $P$-nonclassicality threshold $n_{s}^{\mathrm{P}}$. We focus on the optimal case of a balanced BS, obtaining:

$$
n_{s}^{\text {sep }}=\frac{\left[n_{2}+h\left(n_{s}^{\mathrm{P}}\right)\left(1+2 n_{2}\right)\right]^{2}}{\left(1+2 n_{2}\right)\left[1+2 h\left(n_{s}^{\mathrm{P}}\right)\right]},
$$

where $h\left(n_{s}^{\mathrm{P}}\right)=n_{s}^{\mathrm{P}}+\sqrt{n_{s}^{\mathrm{P}}\left(1+n_{s}^{\mathrm{P}}\right)}$ is a monotonically increasing function of the non-classicality threshold. Even if the two thresholds $n_{s}^{\text {sep }}$ and $n_{s}^{\mathrm{P}}$ now differ, their knowledge enables one, given a known amount of thermal noise in $\hat{a}_{2}$, to estimate the effective $P$-nonclassicality required in $\hat{a}_{1}$, i.e. how much squeezing pump into the BS, in order to get entanglement. When we have a squeezed vacuum state entering the $\mathrm{BS}$ in the mode $\hat{a}_{1}$, namely 
$n_{t}=0$, and $n_{2}$ thermal photons in $\hat{b}$, the separability threshold in Eq. (33) reduces to $n_{2}^{2} /\left(1+2 n_{2}\right)$, independently on $\tau$. It is the value of the curves $n_{s}^{\text {sep }}$ at $n_{t}=0$, as it can be seen from Fig. 8, and moreover it is the same expression as $n_{s}^{\mathrm{nc}}$ with $n_{t}$ replaced by $n_{2}$. Thus, having a squeezed vacuum state in mode $\hat{a}_{1}$ and a thermal state in mode $\hat{a}_{2}$ (characterized by $n_{s}$ squeezed and $n_{2}$ thermal photons, respectively) is equivalent to have a single mode Gaussian state $\varrho\left(n_{s}, n_{2}\right)$ in $\hat{a}_{1}$ and the vacuum in $\hat{a}_{2}$.

Finally, in Fig. 10 we propose the same random plot as in Fig. 5, with the difference that random number of thermal photons is added in the second mode. We can see that the lower branch is substantially unchanged by the presence of thermal noise; even if the entanglement sets later, i.e. for higher amount of squeezing, the relationship with Gaussian discord remains the same. On the other hand, in the remaining accessible region of the plane, compared with Fig. 5, the points are scattered and the sharp pattern is now washed out. On average the distribution drops towards lower values of the discord.

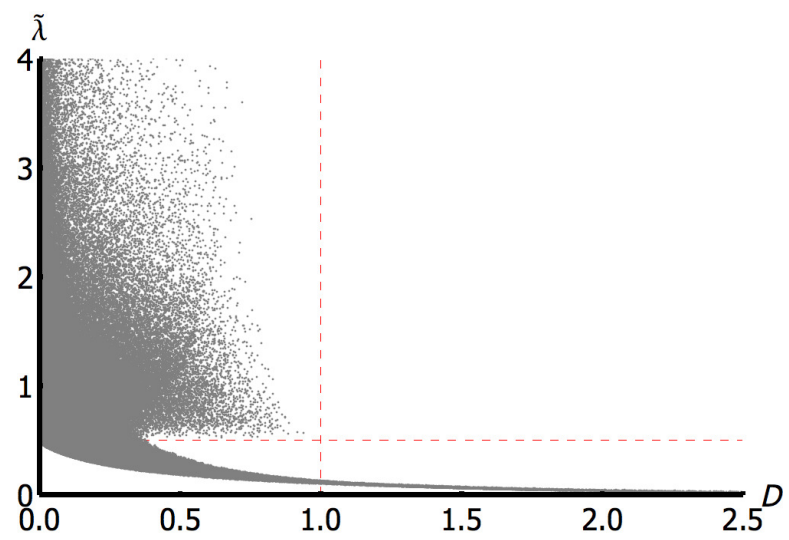

FIG. 10: (Color online) Nonclassicality by mixing with a thermal state: symplectic eigenvalue of the partial transpose $\tilde{\lambda}_{-}$ versus discord for randomly generated input states $\varrho\left(n_{s}, n_{t}\right)$, random values of the transmissivity $\tau$ and randomly generated thermal states $\nu\left(n_{2}\right)$ at the second port of the beam splitter.

\section{Effective non-classicality and non-classical depth}

As said above, in the case in which thermal photons are injected in the second port of the BS, the $P$-nonclassicality is no longer a necessary and sufficient condition to obtain output entanglement. However, remarkably, a quantitative relation between these two notions can still be worked out. In particular, we will now see that the non-classical depth at the input determines the potential of generating entanglement at the output.

Let us first consider the implicit equation defining the separability threshold $\tilde{\lambda}_{-}\left(n_{s}, n_{t}, n_{2}, \tau\right)=1 / 2$, and let us call $\mathcal{E}_{\varrho}(\tau)$ its solution with respect to the number of thermal photons in the second port as a function of $\tau$. It ex- presses (as a function of the input parameters $n_{s}, n_{t}$ ) the number of thermal photons that can enter a BS of transmissivity $\tau$ in the $\hat{a}_{2}$ mode and yield an output entangled state. The explicitly form of $\mathcal{E}_{\varrho}(\tau)$, although analytical, is quite cumbersome and hence has not been reported. If we now perform a maximization over the transmissivity $\tau$ we obtain the following quantity:

$$
\mathcal{E}_{\varrho}=\max _{\tau} \mathcal{E}_{\varrho}(\tau)
$$

We shall refer to this quantity as to the effective nonclassicality of the state $\varrho$ and it embodies the maximum allowed number of thermal photons that can be mixed with $\varrho$ at a BS and still get an entangled output state.

While the non-classical depth is a property of a singlemode state of the field, the effective non-classicality is a property of the two-mode configuration that we are considering. In other words, the effective non-classicality $\mathcal{E}_{\varrho}$ must be intended as an attempt to characterize operationally the non-classical feature of a state. Thus, the relation between $\mathcal{E}_{\varrho}$ and $\mathrm{T}_{m}$, if any, is a priori unclear. Let us stress that the operational interpretation commonly associated to the $\mathrm{T}_{m}$ of a single-mode state $\varrho$ is that it gives the number of thermal photons that have to be statistically mixed with the state $\varrho$ in order to obtain a classical state. In this sense, this operational interpretation of $\mathrm{T}_{m}$ exclusively refers to single-mode states.

In order to clarify the relation between $\mathcal{E}_{\varrho}$ and $\mathrm{T}_{m}$ we notice first that, by means of a numerical maximization, it is possible to show that $\mathcal{E}_{\varrho}$ is always obtained for $\tau=1 / 2$. Thus the balanced BS represents the overall optimal configuration, and in this case the effective non-classicality reads

$$
\mathcal{E}_{\varrho}=\frac{n_{s}-n_{t}+\sqrt{n_{s}\left(1+n_{s}\right)}}{1+2 n_{t}} .
$$

If now we look at the expression of the non-classical depth Eq. (21) and insert it in $\mathcal{E}_{\varrho}$, after some manipulation we find the following relation

$$
\mathcal{E}_{\varrho}=\frac{\mathrm{T}_{m}}{1-2 \mathrm{~T}_{m}}
$$

Thus, the two quantities $\mathcal{E}_{\varrho}$ and $\mathrm{T}_{m}$ which, as said, are defined in reference to different systems, are in fact related via a simple expression. In other words, this endows the non-classical depth with a new operational interpretation: the non-classical depth of a state determines, via Eq. (37), the maximum number of thermal photons that can be mixed with it at a beam splitter without destroying the output entanglement.

\section{CONCLUSIONS}

The quantum-to-classical transition for a single-mode bosonic system may be fully characterized by the properties of its Glauber-Sudarshan $P$-function in the phase 
space. On the other hand, for two-mode states, quantumness may be recognized either by the presence of quantum correlations ( $C$-nonclassicality) or in terms of its phase space distribution ( $P$-nonclassicality). In this paper we have addressed the generation of both types of nonclassicality by the linear mixing of a single-mode Gaussian state with a thermal state at a beam splitter, and have explored in details the relationships between the nonclassical features of the single-mode input and the $P$ - and $C$-nonclassicality of the two-mode outputs.

We have shown that, for mixing with vacuum, a balanced BS is capable of generating $C$-nonclassicality for any input state, contrary to the case of $P$-nonclassicality. In addition, the $C$-nonclassicality increases as the input $P$-nonclassicalitity decreases. These findings clearly confirm in a dynamical setting the inequivalence between these two notions of nonclassicality that was highlighted in Ref. [7] in a geometrical context. We confirm this inequivalence also for mixing with a thermal state, even if more complex behaviors emerge.

In addition, we have shown that input $P$-classicality and output separability single out two thresholds which coincide only for the case of linear mixing with the vacuum, whereas they are connected in a non trivial way for linear mixing with a thermal state. In fact, $P$-classicality at the input, as quantified by the non-classical depth, does determine quantitatively the potential of generating output entanglement. This allows us to provide a new operational interpretation for the non-classical depth: it gives the maximum number of thermal reference photons that can be mixed at a beam splitter without destroying the output entanglement.

By reinforcing quantitatively the inequivalence between $P$ - and $C$-classicality, our results paves the way for analyzing the dynamical relationship between different types of non-classicality in more general contexts.

\section{Acknowledgments}

This work has been supported by MIUR through the FIRB project "LiCHIS" (grant RBFR10YQ3H), by EU through the Collaborative Projects TherMiQ (Grant Agreement 618074) and QuProCS (Grant Agreement 641277) and by UniMI through the H2020 Transition Grant 14-6-3008000-625.
[1] E.P. Wigner, Phys. Rev. 40749 (1932).

[2] R.J. Glauber, Phys. Rev. 131, 2766 (1963).

[3] E. C. G. Sudarshan, Phys. Rev. Lett. 10, 277 (1963) .

[4] L. Mandel, E. Wolf, Optical Coherence and Quantum Optics. (Cambridge University Press, 1995).

[5] R. Horodecki, P. Horodecki, M. Horodecki, and K. Horodecki, Rev. Mod. Phys. 81, 865 (2009).

[6] K. Modi, A. Brodutch, H. Cable, T. Paterek, and V. Vedral, Rev. Mod. Phys. 84, 1655 (2012).

[7] A. Ferraro, M. G. A. Paris Phys. Rev. Lett. 108, 260403 (2012).

[8] V. Chille, N. Quinn, C. Peuntinger, C. Croal, L. Mista Jr., C. Marquardt, G. Leuchs, N. Korolkova, arXiv:1411.6922 [quant-ph].

[9] K. E. Cahill, R. J. Glauber, Phys. Rev. 177, 1857 (1969); 177, 1882 (1969).

[10] S. Olivares, Eur. Phys. J. Special Topics 203, 3 (2012).

[11] C. Weedbrook, S. Pirandola, R. Garc`a-Patròn, N. J. Cerf, T. C. Ralph, J. H. Shapiro and S. Lloyd, Rev. Mod. Phys., 84, 621 (2012).

[12] A. Ferraro, S. Olivares, M. G. A. Paris, Gaussian States in Quantum Information (Bibliopolis, Napoli, 2005).

[13] R. A. Campos, B. E. A. Saleh, M.C. Teich, Phys. Rev. A 40, 1371 (1989); D. Walls and G. Milburn. Quantum optics (Springer Verlag, Berlin. 1994).

[14] P. Meystre, Atom Optics (Springer-Verlag, New York, 2001)

[15] M. J. Woolley, G. J. Milburn, and C. M. Caves, New J. Phys. 10, 125018 (2008).

[16] S.-H. Xiang, W. Wen, Z.-G. Shi, and K.-H. Song, Phys. Rev. A 81, 054301 (2010).

[17] M. Poot, H.S.J. van der Zant, Phys. Rep. 511, 273 (2012).
[18] M. Aspelmeyer, T.J. Kippenberg, F. Marquardt, Rev. Mod. Phys., 86, 1391 (2014).

[19] A. Wallraff et al., Nature 431, 162 (2004).

[20] L. Chirolli, G. Burkard, S. Kumar, and D. P. Di Vincenzo, Phys. Rev. Lett. 104, 230502 (2010).

[21] B. L. Schumaker, Phys. Rep. 135, 317 (1986).

[22] A. Serafini, F. Illuminati, S. De Siena, J. Phys. B 37, L21 (2004).

[23] C. T. Lee, Phys. Rev. A 44, R2775 (1991).

[24] C. T. Lee, Phys. Rev. A 52, 3374 (1995).

[25] M. Takeoka, M. Ban, M. Sasaki, J. Opt. B 4114 (2002).

[26] A. Serafini, M. G. A. Paris, F. Illuminati, and S. De Siena, J. Opt. B 7, R1 (2005).

[27] H. Ollivier and W. H. Zurek, Phys. Rev. Lett. 88, 017901 (2001).

[28] P. Giorda, M. G. A. Paris, Phys. Rev. Lett. 105, 020503 (2010).

[29] G. Adesso, A. Datta, Phys. Rev. Lett. 105, 030501 (2010).

[30] M. Gu, H. M. Chrzanowski, S. M. Assad, T. Symul, K. Modi, T. C. Ralph, V. Vedral, and P. K. Lam, Nat. Phys. 8, 671 (2012).

[31] L. S. Madsen, A. Berni, M. Lassen, and U. L. Andersen, Phys. Rev. Lett. 109, 030402 (2012).

[32] R. Blandino, M. G. Genoni, J. Etesse, M. Barbieri, M. G. A. Paris, P. Grangier, R. Tualle-Brouri, Phys. Rev. Lett 109, 180402 (2012).

[33] S. Pirandola, G. Spedalieri, S. L. Braunstein, N. J. Cerf, and S. Lloyd, Phys. Rev. Lett. 113, 140405 (2014).

[34] R. Simon, Phys. Rev. Lett. 84, 2726 (2000).

[35] A. Peres, Phys. Rev. Lett. 77, 1413 (1996).

[36] G. Vidal, R.F. Werner, Phys. Rev. A 65, 032314 (2002).

[37] M. S. Kim, W. Son, V. Buzek, and P. L. Knight, Phys. 
Rev. A 65, 032323 (2002).

[38] W. Xiang-bin, Phys. Rev. A 66, 024303 (2002).

[39] M.M. Wolf, J. Eisert, and M.B. Plenio, Phys. Rev. Lett. 90, 047904 (2003).

[40] J.K. Asbóth, J. Calsamiglia, and H. Ritsch, Phys. Rev. Lett. 94, 173602 (2005).

[41] S. Olivares and M. G. A. Paris, Phys. Rev. A 80, 032329 (2009).

[42] S. Olivares and M. G. A. Paris, Phys. Rev. Lett. 107, 170505 (2011).
[43] Z. Jiang, M.D. Lang, and C.M. Caves, Phys. Rev. A 88, 044301 (2013).

[44] W. Vogel and J. Sperling, Phys. Rev. A 89, 052302 (2014).

[45] A. Ferraro, L. Aolita, D. Cavalcanti, F. M. Cucchietti, and A. Acín, Phys. Rev. A 81, 052318 (2010).

[46] A. Cazzaniga, S. Maniscalco, S. Olivares, M. G. A. Paris, Phys. Rev. A 88, 032121 (2013). 